\title{
Elevation of miR-302b prevent multiple myeloma cells growth and bone destruction by blocking DKK1 secretion
}

\section{Zheyu Wu}

Wuhan University Zhongnan Hospital https://orcid.org/0000-0002-7517-6153

\section{Yufeng Zhang}

Wuhan University Zhongnan Hospital

\section{Zhiqiang Yang}

Wuhan University Zhongnan Hospital

\section{Yufan Zhu}

Wuhan University Zhongnan Hospital

\section{Yuanlong Xie}

Wuhan University Zhongnan Hospital

\section{Fuling Zhou}

Wuhan University Zhongnan Hospital

Lin Cai ( $D$ orthopedics@whu.edu.cn )

Wuhan University Zhongnan Hospital https://orcid.org/0000-0003-3126-6429

\section{Primary research}

Keywords: Multiple myeloma, Myeloma bone disease, miR-302b, DKK1

Posted Date: October 29th, 2020

DOI: https://doi.org/10.21203/rs.3.rs-98086/v1

License: (a) This work is licensed under a Creative Commons Attribution 4.0 International License. Read Full License

Version of Record: A version of this preprint was published at Cancer Cell International on March 31st, 2021. See the published version at https://doi.org/10.1186/s12935-021-01887-y. 


\section{Abstract}

Background: Myeloma bone disease (MBD) is a severe complication of multiple myeloma (MM) and is mainly attributed to an imbalance between enhanced osteoclast activity and the reducing of osteoblast function. Previous study has indicated that miRNAs serve a vital role in osteogenic differentiation of mesenchymal stromal cells (MSCs) in MM.

Method: MiR-302b belongs to miR-302 cluster, which inhibited the tumor growth and osteolysis in orthotopic osteosarcoma xenograft tumor mouse model in our previous research. In the current study, miR-302b mimics, miR-302b inhibitor and miR-302b NC were transfected into MM1.S and RPMI8226 MM cells. The expression between miR-302b and clinicopathological features in pathological

samples were detected by qPCR. Immunohistochemistry, qPCR and western blot were used to confirm the target relationship between miR-302b and DKK1. Western blotting was employed in analyzing the relationship between miR-302b and the Wnt/ $\beta$-catenin signaling pathway. The effect of miR-302b on osteoblastogenesis was studied using a subperiosteal tumorigenesis model in NOD/SCID nude mice.

Results: Increased miR-302b suppressed cell proliferation and induced the cell apoptosis in RPMI 8226 and MM1.S cells. Targetscan online bioinformatic analysis predicted that miR-302b may bind to 3 'UTR of DKK1 mRNA. Target binding of miR-302b to DKK1 was confirmed by dual-luciferase reporter assay, qPCR, western blot and immunohistochemistry, which indicated that miR-302b caused degradation of DKK1 in RPMI 8226 and MM1.S cells. The model of co-culturing MM cells with preosteoblast MC3T3-E1 cells showed that miR-302b inhibited MM-induced suppression of osteoblast differentiation. Western blotting was used to prove that miR-302b inhibited Wnt/ $\beta$-catenin signaling pathway in MM cells. Micro-CT and immunohistochemistry results showed that miR-302b suppresses the Myeloma bone destruction in vivo.

Conclusion: In conclusion, miR-302b targets DKK1 and inhibited Wnt/ $\beta$-catenin signaling pathway in MM.

\section{Background}

Multiple myeloma (MM) is an incurable uniformly fatal neoplastic disease originating from of B-cell, characterized by malignant clonal hyperplasia of plasma cells in bone marrow, leading to monoclonal immunoglobulin (paraprotein), renal dysfunction, hypercalcemia and lytic lesions in bone[1, 2]. In addition, myeloma bone disease (MBD) which affects almost 70\% of myeloma patients at the time of diagnosis, is mainly caused by an imbalance of the bone remodeling process in the bone marrow microenvironment[3]. Bone destruction in MBD is usually attributed to over-activation of osteoclastic activity and inhibition of osteoblastogenesis[4, 5].

Complex cell-cell interactions among MM tumor cells are essential for MBD [6, 7]. Previous studys have indicated that MM cells could impair the normal physiological function of bone marrow derived stromal cells (BMSCs), osteoclasts and osteocytes[8-10]. Several cytokines and growth factors secreted by BMSCs, such as Wnt inhibitors, interleukin-3 (IL-3), tumor necrosis factor alpha (TNF-a) and transforming 
growth factor $\mathrm{b}$ (TGF-b), were overproduced by MM cells, which are considered as the most important factors for the osteoblast dysfunction of myeloma patients[11-13]. However, the mutual regulation mechanisms between MM cells and osteoblast from different differentiation stages haven't been revealed.

MicroRNAs (miRNAs) are small non-coding single-stranded RNA molecules with a length of about 22 nucleotides, which are widely involved in post-transcriptional regulation of eukaryotic gene expression[14, 15]. Multiple miRNAs including miR-29b, miR-21, miR-15a, miR-192 and miR-215 have been identified to be implicated in the development of $\mathrm{MM}$ and the regulation of bone marrow microenvironment[16-19]. A study reported that overexpression of miR-29b reduced the stimulation response of osteoclasts to RANKL through the target gene c-FOS and the secretion of osteolytic enzymes. Leone et al. showed that miR-21 promoted the progression of MM cells through inhibition of OPG[20]. Furthermore, miRNAs have been shown to involve in the differentiation process of preosteoblast. Also, the osteogenic was regulated by several miRNAs, including miR-135b, miR-22, miR-140[21, 22].

MiR-302b belongs to miR-302 cluster, which is located at 4q25 chromosome region and serves as a tumor suppressor in osteosarcoma, gastric cancer, hepatocellular carcinoma and bladder cancer[23].Previous studies showed that miR-302b inhibited the tumor growth and osteolysis in orthotopic osteosarcoma xenograft tumor mouse model made by injecting osteosarcoma cells subperiosteally into the right proximal lateral tibia[24]. However, the role of miR-302b in multiple myeloma and MMD is unclear. Here, we found that miR-302b was lower expressed in the bone marrow of patients with multiple myeloma than normal people. Up-regulation of miR-302b inhibited the proliferation and induced the cell apoptosis in MM cell lines, and inhibited MM-induced suppression of osteoblast differentiation. We also revealed that miR-302b may extenuate MM cells induced bone destruction through targeting DKK1 expression and secretion.

\section{Methods}

\section{Cell Culture and Osteogenic Differentiation}

The human multiple myeloma cell lines RPMI8226, and MM1.S were cultured in RPMI1640 supplemented with $10 \%$ fetal bovine serum at $37^{\circ} \mathrm{C}$ in a $5 \% \mathrm{CO} 2$ atmosphere, the medium was renewed every three days. MC3T3-E1 were cultured in DMEM supplemented with $10 \%$ fetal bovine serum and $1 \%$ L-glutamine at $37^{\circ} \mathrm{C}$ in a $5 \% \mathrm{CO} 2$ atmosphere. 24-well multiwell insert System with $1.0 \mu \mathrm{m}$ pore PET Membrane was used for establishing cell co-culture model, MC3T3-E1 were seeded in 12-well plates. MC3T3-E1 were seeded in the lower chamber and MM cells were seeded in the upper chamber, respectively. After coculture for $24 \mathrm{~h}$, the MM cells were removed and MC3T3-E1 were cultured with renewed medium for further study. For osteogenic differentiation, MC3T3-E1 were cultured in a-MEM containing 50uM ascorbic acid 2-phosphate, $5 \mathrm{mM} \beta$-glycerophosphate, and $100 \mathrm{nM}$ dexamethasone.

\section{Trantsient transfection}


MiR-302b mimics and inhibitor were obtained from RiboBio. For the miR-302b mimic and inhibitor transfection, MM1.S and RPMI8226 cells at a concentration of $2 \times 104$ cells/well were incubated in 6-well plates for $24 \mathrm{~h}$. Then, the cells were transfected with $50 \mathrm{nM}$ miR-302b mimic or $100 \mathrm{nM}$ inhibitor in OptiMEM medium without FBS addition of Lipofectamine 3000, according to the manufacturer's protocol.

\section{MTT assay}

MTT assay was used to detect the MM cell viability. Multiple myeloma cells(5000 cells per well) were seeded in 96-well plates in RPMI1640 medium. $24 \mathrm{~h}$ after the cells were seeded, trantsient transfection was performed according to the manufacturer's protocol. After $24 \mathrm{~h}, 48 \mathrm{~h}$ and $72 \mathrm{~h}$ transfection, $10 \mu \mathrm{l}$ of $5 \mathrm{mg} / \mathrm{ml}$ MTT solution was added to each well and then the cells were incubated for $4 \mathrm{~h}$ at $37^{\circ} \mathrm{C}, 5 \%$ CO2. Cells in 96 -well plates were centrifuged at $1,000 \times \mathrm{g}$ for $10 \mathrm{~min}$ and then the medium was removed. $100 \mu \mathrm{L}$ DMSO was added to each well and the plates were shaked on the oscillator dissolve for 10 min to dissolve the formazan crystals. Optical density (OD) of each well was measured at $570 \mathrm{~nm}$ using a microplate Reader(PE Enspire).

\section{Flow cytometry detection:}

Annexin V-FITC Apoptosis Detection Kit(CA1020) was obtained from Beijing Solarbio Science \& Technology Co., Ltd. (China). Briefly, $5 \times 10^{5}$ sample cells were collected and washed with $4{ }^{\circ} \mathrm{C}$ PBS three times, and resuspended in $200 \mu$ l Binding Buffer. In the darkness, $10 \mu$ l Annexin V-FITC and $10 \mu \mathrm{II}$ were mixed and added into the cell suspension to react for 15 minutes at room temperature. Then $300 \mu \mathrm{l}$ Binding Buffer was added and the samples were detected by flow cytometry (Cytoflex, Beckman) within 1 hour.

\section{Alizarin red staining and quantitative analysis of mineralization}

After 10 days osteogenic differentiation, MC3T3-E1 cells were washed twice with PBS and then fixed with $4 \%$ paraformaldehyde for $5 \mathrm{~min}$. Cell plates were washed with deionized water twice and then stained with 1\% Alizarin ( $\mathrm{pH}$ 4.1) Red S solution for 20 min. The deposited Alizarin Red S solution in each well was collected and mixed with $10 \%$ cetylpyridinium chloride (Sigma-Aldrich) and evaluated for absorbance at $560 \mathrm{~nm}$. Then the plates were washed with water three times and were photographed under light microscope. The orange and red positions were identified as Calcium deposits.

\section{Quantitative Real-time PCR(qPCR)}

Total RNA was extracted from cells by using TRIzol Regent (Invitrogen). The RNA was reverse transcribed using a Transcriptor Universal cDNA Master (Roche) according to the manufacturer's instructions. Realtime PCR was performed as manufacturer's instructions (Applied Biosystems). PCR amplification was carried out on CFX Connect Real-Time PCR Detection System(Bio-Rad, Richmond, CA). Primer sequences: miR-302b forward 5'-ATCCAGTGCGTGTCGTG-3', reverse 5'-TGCTTAAGTGCTTCCATGTT-3';. U6 was used as an internal control of miR-302b.U6 forward 5'-CTCGCTTCGGCAGCACATATACT-3', reverse 5'ACGCTTCACGAATTTGCGTGTC-3'. Other target genes used $\beta$-actin as an internal control. All the experiments were repeated three times. 


\section{Dual-luciferase reporter assay}

The wild-type (WT) sequence of DKK1 mRNA 3'UTR(WT-DKK1-3'UTR) and mutation (MUT) sequence of DKK1 mRNA 3'UTR with deletion of the miR-302b binding sites(MUT-DKK1-3'UTR) were designed consequently. Luciferase report vectors were constructed. For dual luciferase reporter detection, miR-302b mimics was co-transfected with wide type or mutant pmirGLO-3'UTR vectors into RPMI 8226 or MM1.S cells, respectively. After $48 \mathrm{~h}$ of transfection, the fluorescence activity was detected using Dual-Gloß Luciferase Assay Reagent (Promega, USA) following the standard protocol on GloMax® 20/20 Luminometer (Promega, USA).

\section{Western blot detection}

Cells were harvested and lysed in the RIPA buffer (Cell Signaling Technology, 9806), After the lysates were sonicated, the protein concentration was determined by the BCA assay. Proteins were separated by SDSPAGE and transferred electrophoretically onto a PVDF membrane (Millipore, IPVH00010). Each antibody incubation was performed overnight at $4{ }^{\circ} \mathrm{C}$ followed by the secondary antibody treatment for $1 \mathrm{~h}$ at room temperature. We used primary antibodies against Bcl-2(Abcam,1:5000), Bax(Abcam®1:3000), and DKK1(Abcam®1:3000), Runx2(Abcam $\otimes 1: 3000) \otimes \beta$-catenin(Abcam $\otimes 1: 5000)$. We used $\beta$ actin(Abcam $\mathbb{1}: 2000)$ as an internal control for all analyses. Immunoblotting was visualized using ECL system and analyzed using Image-Pro Plus 6.0.

\section{MM bone destruction mouse model}

The animal study was approved by the ethical committee of Zhongnan Hospital of Wuhan University. All of the procedures for the animals in this study were performed in accordance with the Declaration of Helsinki. MM1.S cells $(0.5-1 \times 106$ cells $/ 100 \mu$ in PBS) were injected into the femur of NOD/ SCID (4 weeks, female) mice, PBS was injected into the contralateral side as a control. When the weight of mice droped significantly, $40 \mathrm{ul} \mathrm{hsa-miR-302b}$ agonist $(10 \mathrm{mM})$ with multiple myeloma of NOD/SCID mice were injected in the femurs every $3 \mathrm{~d}$ in 2 weeks, then execute mice and isolation of femurs in mice were used to detect bone destruction by Micro CT. All animal experiments were performed with approval from the Animal Study Committee of WuHan University and conformed to the relevant guidelines and legislations.

\section{Micro CT detection}

Micro-CT analysis was performed using R_mCT2 (SkyScan1278, Bruker) with an isometric resolution of $40 \mu \mathrm{m}$, or ScanXmateL090 with an isometric resolution of $12 \mu \mathrm{m}$. The micro-CT files were reconstructed as TIFF images and transferred to TRI/FCS-BON for the quantitative analysis. We measured the mineralized tissue volume using a calibration curve obtained from MicroCT Bar Pattern NANO Phantom.

\section{Histology and Immunohistochemistry.}

To perform histological analyses of induced osteoblastic lesions, H\&E and von Kossa staining were performed using paraffin and undecalcified sections respectively. Immunohistochemistry was performed on frozen sections as previously mentioned. Sections were examined using a fluorescence microscope (TE2000U + NT88, NIKON) or a confocal microscope ( $\mu$ Surf, Nanofocus). 


\section{Statistical Analyses.}

All the data were presented as the means \pm SEM. We performed parametric statistics by Student's $t$ test, one-way ANOVA with Tukey's HSD test, nonparametric statistics by the Mann-Whitney U test, or the Kruskal-Wallis test using $R$ v3.3.1. The values were considered significant at $P<0.05$. The results were representative of more than three individual experiments.

\section{Clinical MM specimens}

The study was approved by the Ethics Committee of Zhongnan Hospital of Wuhan University and conducted in accordance with the Declaration of Helsinki. Twelve pairs of MM patients and healthy individuals' bone marrow were collected from the Department of Orthopedics of Zhongnan Hospital of Wuhan University from February 2019 to July 2020. All of the specimens were obtained with the patients' informed consent. All tumor samples were pathologically diagnosed as MM according to the World Health Organization (WHO) Classification of myeloid and lymphoid neoplasms.

\section{Results}

\section{Elevation of miR-302b suppressed cell proliferation and induced the cell apoptosis in RPMI 8226 and MM1.S cells.}

In order to explore the effect of miR-302b on multiple myeloma (MM), bone marrow of $12 \mathrm{MM}$ patients and 12 healthy individuals were collected, and the results showed that miR-302b was lower expressed in bone marrow of multiple myeloma patients compared with the healthy subjects. MiR-302b in MM cells (RPMI 8226 and MM1.S) was elevated with transient transfection of miR-302b mimics respectively (Fig. 1A-C). MTT assay results showed that elevation of miR-302b significantly suppressed the cell proliferation at $24,48,72 \mathrm{~h}$ after the transient transfection of miR-302b mimics in RPMI 8226 and MM1.S cells. The early and late stage apoptotic rate was significantly promoted in miR-302b mimics group compared with the control group in RPMI 8226 and MM1.S cells (Fig. 1D-E). Relative protein expression of $\mathrm{Bcl}-2$ was promoted, and Bax was inhibited in miR-302b mimics group compared with the control group in RPMI 8226 and MM1.S cells (Fig. 1F). The gain of function study showed that elevation of miR$302 \mathrm{~b}$ suppressed cell proliferation in RPMI 8226 and MM1.S cells, which may due to the induced cell apoptosis by miR-302b.

\section{Inhibition of miR-302b induced cell apoptosis and arrested the cell apoptosis in RPMI 8226 and MM1.S cells.}

MiR-302b was down-regulated through transient transfection of miR-302b inhibitor for further study, which evaluated the loss of function study on miR-302b in RPMI 8226 and MM1.S cells. The speed of RPMI 8226 and MM1.S cells' proliferation promoted at 24, 48, $72 \mathrm{~h}$ after the transient transfection in miR$302 \mathrm{~b}$ inhibitor group compared with the control group respectively (Fig. 2A-C). The early and late stage apoptotic rate was significantly decreased in the miR-302b inhibitor group compared with the control 
group in RPMI 8226 and MM1.S cells (Fig. 2D-E). Suppression of miR-302b inhibited relative protein expression of $\mathrm{Bcl}-2$ and promoted Bax in RPMI 8226 and MM1.S cells (Fig. 2F). The loss of function study showed that suppression of miR-302b promoted cell proliferation in RPMI 8226 and MM1.S cells

\section{3. miR-302b targeted DKK1 in RPMI 8226 and MM1.S cells.}

Targetscan online bioinformatic analysis predicted that miR-302b may bind to 3'UTR of DKK1 mRNA. A dual-luciferase reporter assay was applied to clarify whether the 3'-UTR of DKK1 mRNA was directly targeted by miR-302b. The putative wide type binding site of DKK1 mRNA 3'-UTR was cloned into the pmirGLO vector. For dual luciferase reporter detection, miR-302b mimics were co-transfected with wild type or mutant pmirGLO-3'UTR vectors into RPMI 8226 or MM1.S cells (Fig. 3A). As shown in Fig, the relative luciferase activity was significantly suppressed by transfecting miR-302b mimics when the wildtype 3'-UTR of DKK1 was respectively present in RPMI 8226 or MM1.S cells (Fig. 3B-C). Whereas miR302b mimics exerted no significant change of luciferase activity in RPMI 8226 and MM1.S cells cotransfected with mutant type 3'-UTR of DKK1. Furthermore, miR-302b mimics inhibited the protein expression of DKK1 in RPMI 8226 and MM1.S cells (Fig. 3D-E). These results suggest that miR-302b may target 3'-UTR of DKK1 mRNA in RPMI 8226 and MM1.S cells.

\section{4. miR-302b inhibited MM-induced suppression of osteoblast differentiation}

A model of co-culturing MM cells with preosteoblast MC3T3-E1 cells was applied to further elucidate the potential role of miR-302b in MM bone disease. When co-cultured with RPMI 8226 or MM1.S, mineralization capacity of MC3T3-E1 cells were suppressed compared with the cells without co-culture in blank group (Fig. 4A-B). Mineralization capacity of MC3T3-E1 co-cultured with miR-302b up-regulated RPMI 8226 or MM1.S was stronger than that of MC3T3-E1 co-cultured with miR-302b normally expressed MM cells. DKK1 protein in the supernatant of MC3T3-E1 cells was detected by ELISA. Both RPMI 8226 and MM1.S promoted the DKK1 protein content in the supernatant of MC3T3-E1 at $24 \mathrm{~h}$ after the coculture when compared with the blank group (Fig. 4C). Furthemore, DKK1 protein content in the supernatant of MC3T3-E1 co-cultured with miR-302b up-regulated RPMI 8226 or MM1.S was more than that of the control group. RT-PCR results showed that both RPMI 8226 and MM1.S suppressed the mRNA expression of Collagen I of MC3T3-E1 at $24 \mathrm{~h}$ after the co-culture. And the Collagen I mRNA expression of MC3T3-E1 co-cultured with miR-302b was lower expressed in RPMI 8226 or MM1.S, which was compared with the MC3T3-E1 cells co-cultured with miR-302b normally expressed MM cells respectively (Fig. 4D). Taken together, our study proved that MM cells could impair osteogenic differentiation of MC3T3-E1 cells, and miR-302b could remedy the MM-induced suppression of MC3T3-E1 cells differentiation.

\section{5. miR-302b inhibited Wnt/ $\beta$-catenin signaling pathway in MM cells}

To better understand whether Wnt/ $\beta$-catenin signaling pathway involves in the regulation of $\mathrm{MM}$ induced by miR-302b, miR-302b mimics (mimics)/miR302b mimics-control (mimics ctrl) and DKK1 overexpression vectors (DKK1)/negative control vectors (DKK1 ctrl) were cotransfected into RPMI 8226 and MM1.S cells. We detected the relative protein expression of markers by western blot. The expression of DKK1 and GSK3 was markedly down-regulated in miR-302b overexpressed in RPMI 8226 and MM1.S 
cells (Fig. 5B). Furthemore, miR-302b negative control induced significant inhibition of LRP6 and Wnt3a in both RPMI 8226 and MM1.S cells (Fig. 5B-C).These results demonstrated that Wnt/ $\beta$-catenin signaling pathway involved in the miR-302b mediated regulatory effect.

\section{6. miR-302b suppresses the Myeloma bone destruction in vivo}

To investigate whether miR-302b can suppress the MM cells induced bone destruction in vivo, MM1.S cells transfected with miR-302b mimics or miR-302b negative control were injected into the femur of the NOD/SCID nude mice respectively. 8 weeks after the injection, the mice were dissected and the femurs were detached and detected with micro-CT. The results showed that the bone volume and trabecular number of the mice femur were more in miR-302b mimics group compared with miR-302b negative group (Fig. 6A). And immunohistochemistry results showed that DKK1 expression intensity was lower in miR$302 \mathrm{~b}$ mimics transfection group compared with the miR-302b negative control transfection group. It suggested that overexpression of miR-302b suppressed the MM bone destruction in vivo. And 4 weeks after the molding operation, DKK1 recombinant protein was locally injected into the femur of MM bone destruction model mice induced by MM1.S cells transfected with miR-302b mimics. Micro-CT results showed that DKK1 offset the suppression of miR-302b mimics on bone volume and trabecular number of the mice femur (Fig. 6B-C). Furthermore, DKK1 recombinant protein enhanced the loss of bone volume and trabecular number in mimics NC DKK1 group compared with the control group.

\section{7. miR-302b was lower expressed in MM patient bone marrow.}

Twelve pairs of MM patients' bone marrow was collected and RT-PCR results showed that miR-302b was lower expressed in bone marrow of MM patient compared with the healthy individuals. And immunohistochemistry results showed that DKK1 expression intensity was lower in bone marrow of MM patients compared with the healthy individuals (Fig. 6A-B).

\section{Disscussion}

MBD is progressive with the clinical characteristics of osteolytic lesions, pathological fracture, osteoporosis and intractable bone pain. Under the invasiveness of MM cells, physiological coordination between osteoblasts and osteoclasts is destroyed, resulting in osteogenic-osteoclast coupling imbalance[25]. Consequently, understanding the interactions between MM cells and preosteoblasts within the bone marrow microenvironment has a potential to uncover more effective treatments. Several miRNAs are involved in regulating proliferation of MM cells and osteogenic differentiation of preosteoblast, but still, the role of miRNAs in MBD isn't completely uncovered.

Present study showed that miR-302b was lower expressed in MM patients' bone marrow, and overexpression of miR-302b impeded the cell proliferation and induced apoptosis of MM cells. Both RPMI 8226 and MM1.S could suppress the osteogenic differentiation of preosteoblast MC3T3-E1. Overexpression of miR-302b in MM cells offset the inhibition of MM cells on osteogenic differentiation of preosteoblast MC3T3-E1. And MM cells overexpressed with miR-302b induced lower loss of bone volume and trabecular number compared with the MM cells with normal expression of miR-302b. Previous 
studies have shown that miR-302b was lower expressed in osteosarcoma, liver cancer and prostate cancer and elevation of miR-302b inhibited the biological activity of these cancer cells[26-28]. It has been demonstrated that expression level of miR-221-5p was significantly lower in MBD-MSCs compared with that in N-MSCs, and knockdown of miR-221-5p could promote osteogenic differentiation in MBDMSCs[25]. Thus miR-302b may suppress MM cells proliferation and MM cells induced bone destruction.

A dual-luciferase reporter assay demonstrated that miR-302b targeted 3'-UTR of DKK1 mRNA in multiple myeloma cells. MiR-302b also inhibited secretion of DKK1 in multiple myeloma cells. DKK1 is a secreted protein that binds to the Wnt receptor LRP5 on the cell membrane and inhibits the Wnt/ $\beta$-catenin signaling pathway associated with osteogenic differentiation. DKK1 expression in bone marrow and serum of MM patients with osteolytic destruction was significantly higher than that of MM patients without osteolytic destruction, and the expression level of DKK1 was correlated with the degree of osteolytic destruction in the MRI imaging of MM patients. Tian et al found that the anti-DKK1 vaccine triggered $\mathrm{CD} 4+$ and $\mathrm{CD} 8+\mathrm{T}$ cell responses to protect nude mice from MM invasion. Therefore, miR-302b may rescue the inhibition of MM cells on osteogenic differentiation of MC3T3-E1 cells through suppressing DKK1 secretion.

As shown by a series of experiments, miR-302b is downregulated in MM, and overexpression of miR-302b impeded MM proliferation and metastasis both in vitro and in vivo. DKK1 as one of the four members of extracellular Wnt inhibitors family, serves as the potential target of miR-302b in MM.

\section{Conclusion}

In conclusion, we demonstrated that miR-302b plays an important role in the development of MM. Our study concluded that the overexpression of miR-302b prevent multiple myeloma cells growth and bone destruction by down-regulated DKK1 secretion. Our findings not only improve the understanding of how miR-302b modulates the Wnt/ $\beta$-catenin signaling pathway in MM, but also offer a potential prognostic marker and a therapeutic target for the treatment of $\mathrm{MM}$.

\section{Declarations}

\section{Acknowledgments}

The authors thank Profs. Ansong Ping and Jun Lei from the Department of Orthopedics, Zhongnan Hospital of Wuhan University, China for assisting in tissue collection and experimental design. Thanks Dr Zhang Wenjun from Seattle University for assisting in checking the English grammar and spelling.

\section{Funding}

This study was funded by the Zhongnan Hospital of Wuhan University Science, Technology and Innovation Seed Fund (Project No. znpy2018034) and National Natural Science Foundation of China (Grant No. 81870162). 
Ethics approval and consent to participate

The study was approved by the Ethics Committee of Zhongnan Hospital of Wuhan University and was conducted in accordance with the Declaration of Helsinki. All of the specimens were obtained with the patients' informed consent.

\section{Author contributions}

Wu Zheyu, Zhang Yufeng and Yang Zhiqiang performed the experiments, wrote the manuscript, and prepared the figures. Zhu Yufan analyzed the data. Cai Lin and Zhou Fuling conceived the experiments.

Availability of data and materials

All the data and materials were available under the agreement of the authors

Consent for publication

Not applicable.

\section{Competing interests}

The authors declare that they have no competing interests.

\section{References}

1. Manier S, Salem KZ, Park J, Landau DA, Getz G, Ghobrial IMJNRCO: Genomic complexity of multiple myeloma and its clinical implications. 2016.

2. Anderson, K. CJCCR: Progress and Paradigms in Multiple Myeloma. 2016, 22(22):5419-5427.

3. Garayoa M, Garcia JL, Santamaria C, Garcia-Gomez A, Blanco JF, Pandiella A, Hernández JM, Sanchez-Guijo FM, Del Ca?Izo MC, Gutiérrez NCJL: Mesenchymal stem cells from multiple myeloma patients display distinct genomic profile as compared with those from normal donors. 2009, 23(8):1515-1527.

4. Terpos E, Dimopoulos MAJJoBO: Myeloma bone disease: pathophysiology and management. 2013, 2(2):59.

5. Vyzoukaki, R., ·Tsirakis, G., ·Pappa, C. A, ·Devetzoglou, M., ·Tzardi, Immunol MJIAA: The Impact of Mast Cell Density on the Progression of Bone Disease in Multiple Myeloma Patients. 2015.

6. Delgado-Calle J, Anderson J, Cregor MD, Condon KW, Kuhstoss SA, Plotkin LI, Bellido T, Roodman GDJL: Genetic deletion of sost or pharmacological inhibition of sclerostin prevent multiple myelomainduced bone disease without affecting tumor growth. 2017.

7. Negaard HFS, Iversen N, Bowitz-Lothe IM, Sandset PM, Leukemia POIJ: Increased bone marrow microvascular density in haematological malignancies is associated with differential regulation of angiogenic factors. 2009, 23(1):162-169. 
8. Raje N, Roodman GDJCCR: Advances in the Biology and Treatment of Bone Disease in Multiple Myeloma. 2011, 17(6):1278-1286.

9. Brady JJB: Bone Turnover Biomarkers Are Useful In Monitoring Myeloma Bone Disease and As Early Predictor Biomarkers For Relapse Disease In Multiple Myeloma. 2013, 122.

10. Durie, Brian GM, Ness V, Brian, Ramos, Christine, Stephens, Owen, Haznadar, Blood MJ: Genetic Polymorphisms Identify the Likelihood of Bone Disease in Myeloma: Correlations with Myeloma Cell DKK1 Expression and High Risk Gene Signatures.

11. Marco R, Maria TDM, Eugenio M, Marzia L, Antonietta R, Anna G, Gabriella M, Pierfrancesco T, Michele CJCCDT: Molecular Targets for the Treatment of Multiple Myeloma. 2012, 12(7):--

12. Morgan GJ, Walker BA, Davies FEJNRC: The genetic architecture of multiple myeloma. 2012, 12(5):335-348.

13. Misso G, Zappavigna S, Castellano M, De Rosa G, Di Martino MT, Tagliaferri P, Tassone P, Caraglia MJEOoBT: Emerging pathways as individualized therapeutic target of multiple myeloma. 2013, 13(S1):S95-S109.

14. Tesfaye, Worku, Zia, Rehman, Hira, Talpur, Dinesh, Bhattarai, Farman, Sciences UJIJoM: MicroRNAs: New Insight in Modulating Follicular Atresia: A Review. 2017.

15. Amodio N, Martino MTD, Foresta U, Leone E, Lionetti M, Leotta M, Gullà AM, Pitari MR, Conforti F, Rossi MJCD et al: miR-29b sensitizes multiple myeloma cells to bortezomib-induced apoptosis through the activation of a feedback loop with the transcription factor Sp1. 2012, 3(11):e436.

16. Martino MTD, Leone E, Amodio N, Foresta U, Lionetti M, Pitari MR, Cantafio MEG, Gulla A, Conforti F, Morelli EJCCR: Synthetic miR-34a Mimics as a Novel Therapeutic Agent for Multiple Myeloma: In Vitro and In Vivo Evidence. 2012.

17. Pichiorri F, Suh S-S, Rocci A, Luca LD, Taccioli C, Santhanam R, Zhou W, Jr. DMB, Hofmainster C, Alder $\mathrm{H}$ et al: Downregulation of p53-inducible microRNAs 192, 194, and 215 Impairs the p53/MDM2 Autoregulatory Loop in Multiple Myeloma Development. 2010.

18. Chun-Yan S, Xiao-Mei S, You Q, Zhang-Bo C, Lei C, Li-Sha A, Lu Z, Yu HJC: miR-15a and miR-16 affect the angiogenesis of multiple myeloma by targeting VEGF. 2013(2):426-435.

19. Khalife J, Ghose J, Martella M, Viola D, Pichiorri FJJI: MiR-16 regulates crosstalk in NF-kB tolerogenic inflammatory signaling between myeloma cells and bone marrow macrophages. 2019, 4(21).

20. E., Leone, E., Morelli, M., T., Di, Martino, N., Research AJCC: Targeting miR-21 Inhibits In Vitro and In Vivo Multiple Myeloma Cell Growth. 2013.

21. Umezu T, Tadokoro H, Azuma K, Yoshizawa S, Ohyashiki JHJB: Exosomal miR-135b shed from hypoxic multiple myeloma cells enhances angiogenesis by targeting factor-inhibiting HIF-1. 2014, 124(25):3748.

22. Tardif G, Hum D, Pelletier JP, Duval N, Martel-Pelletier JJBMD: Regulation of the IGFBP-5 and MMP13 genes by the microRNAs miR-140 and miR-27a in human osteoarthritic chondrocytes. 2009, 10 . 
23. Yadav S, Pandey A, Shukla A, Talwelkar SS, Kumar A, Pant AB, Parmar DJJoBC: miR-497 and miR$302 \mathrm{~b}$ regulate ethanol-induced neuronal cell death through BCL2 protein and cyclin D2. 2011, 286(43):37347-37357.

24. Xie Y, Sun W, Deng Z, Zhu X, Hu C, Cai LJSR: MiR-302b Suppresses Osteosarcoma Cell Migration and Invasion by Targeting Runx2. 2017, 7(1):13388.

25. Diao Y, Ma Q, Cui F, Zhong YJJoBMRPA: Human umbilical cord mesenchymal stem cells: Osteogenesis in vivo as seed cells for bone tissue engineering. 2010, 91A(1):123-131.

26. Zhang $Y$, Hu H, Song L, Cai L, Wei R, Jin WJTL: Epirubicin-mediated expression of miR-302b is involved in osteosarcoma apoptosis and cell cycle regulation. 2013, 222(1):1-9.

27. Yadav S, Pandey A, Shukla A, Talwelkar SS, Kumar A, Pant AB, Parmar DJJoBC: miR-497 and miR302b Regulate Ethanol-induced Neuronal Cell Death through BCL2 Protein and Cyclin D2. 2011.

28. Li J, Yu J, Zhang H, Wang B, Guo H, Bai J, Wang J, Dong Y, Zhao Y, Wang YJCP et al: ExosomesDerived MiR-302b Suppresses Lung Cancer Cell Proliferation and Migration via TGF $\beta R I I$ Inhibition. 2016, 38(5):1715-1726.

\section{Figures}




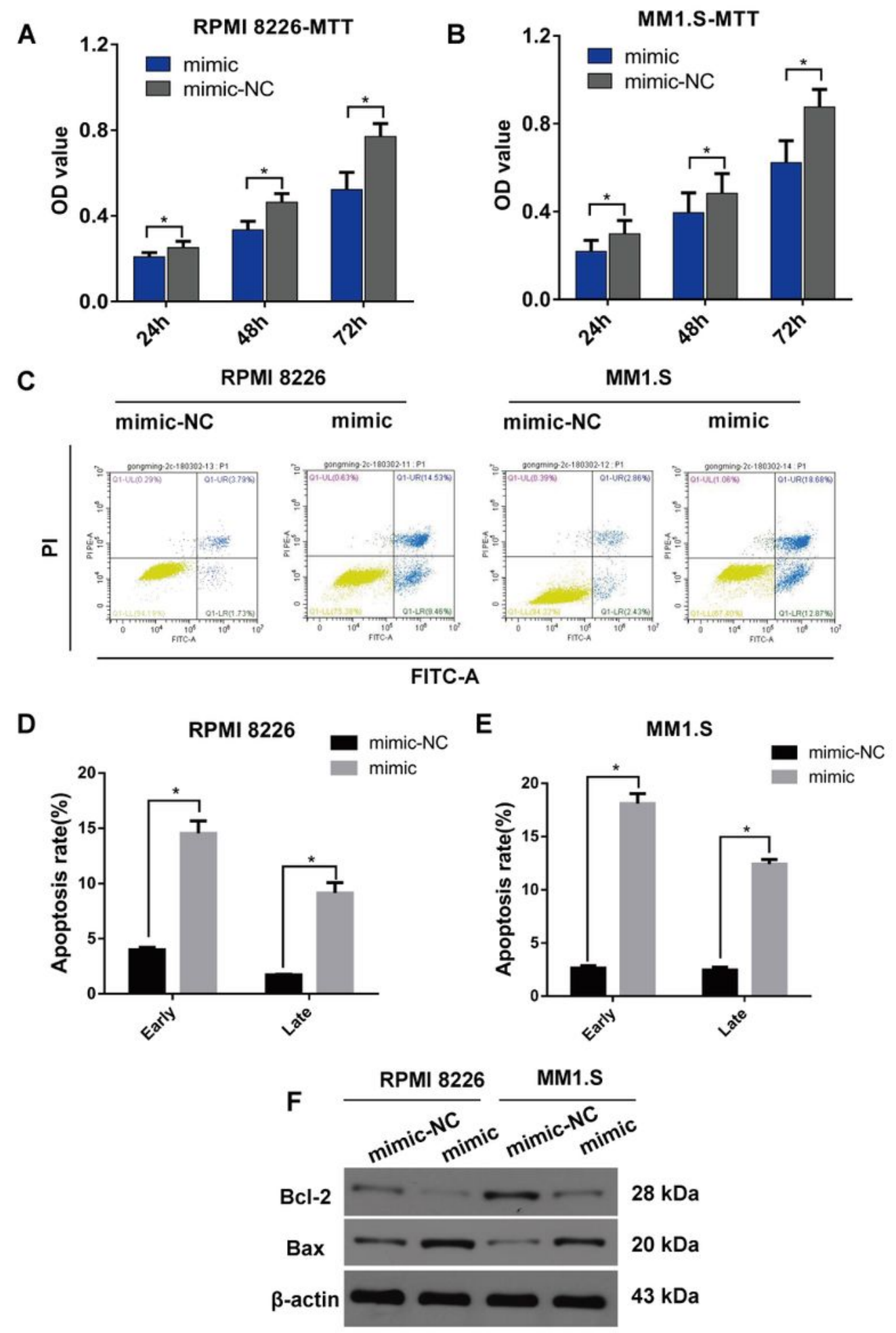

\section{Figure 1}

Overexpression of miR-302b regulated cell proliferation and apoptosis in vitro. (A-B) OD value of RPMI 8226 and MM1.S cells was measured by MTT assay at 24, 48, 72h after the transient transfection of miR302b mimics. (C) Representative images of the apoptosis detection. (D) Cartogram of the apoptosis rate of cells in each group. (E) Expression of relative protein in RPMI 8226 and MM1.S cells. 


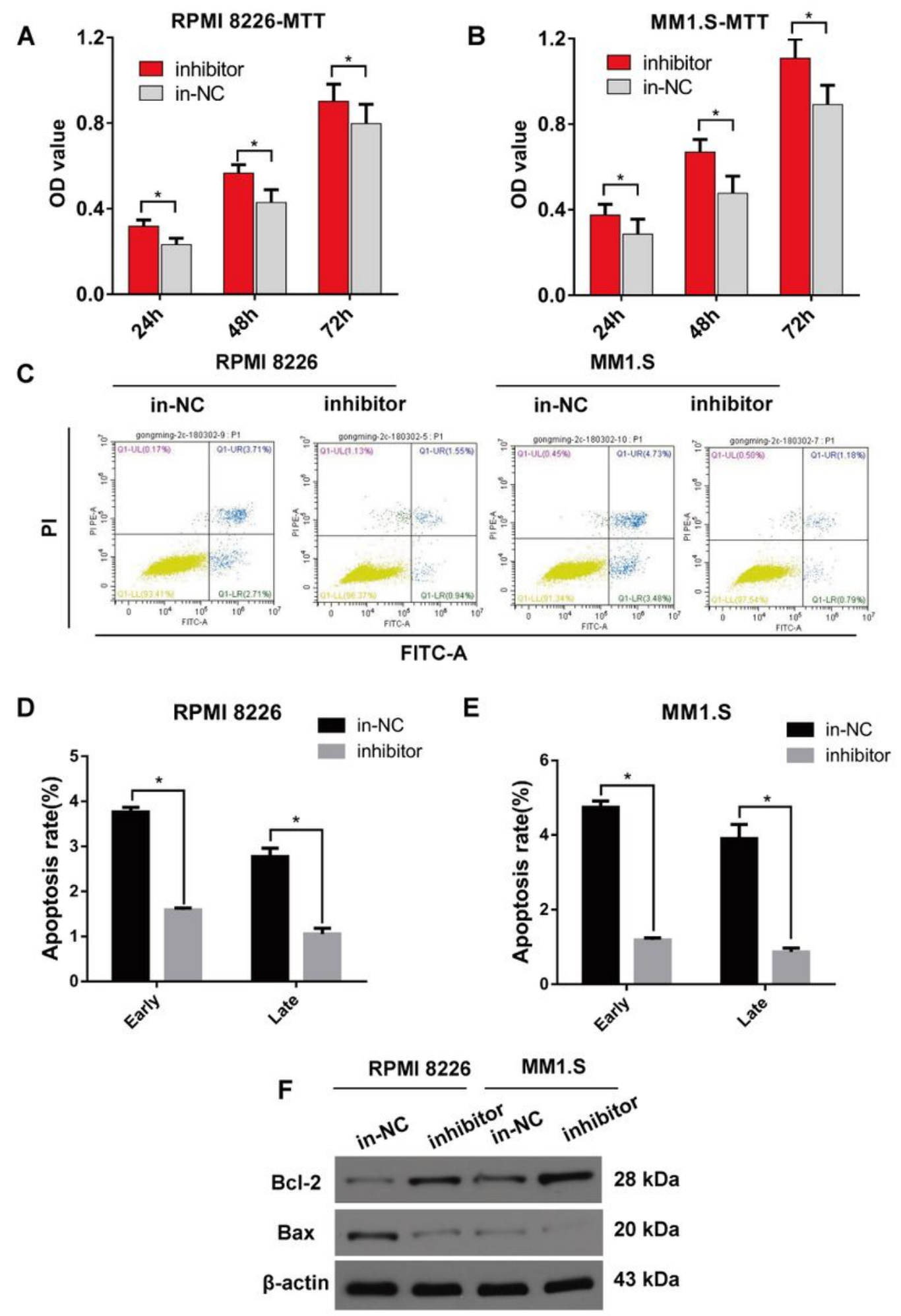

Figure 2

Inhibition of miR-302b regulated cell proliferation and apoptosis in vitro. (A-B) OD value of RPMI 8226 and MM1.S cells was measured by MTT assay at 24, 48, 72h after the transient transfection of miR-302b inhibitors. (C) Representative images of the apoptosis detection. (D) Cartogram of the apoptosis rate of cells in each group. (E) Expression of relative protein in RPMI 8226 and MM1.S cells. 
A
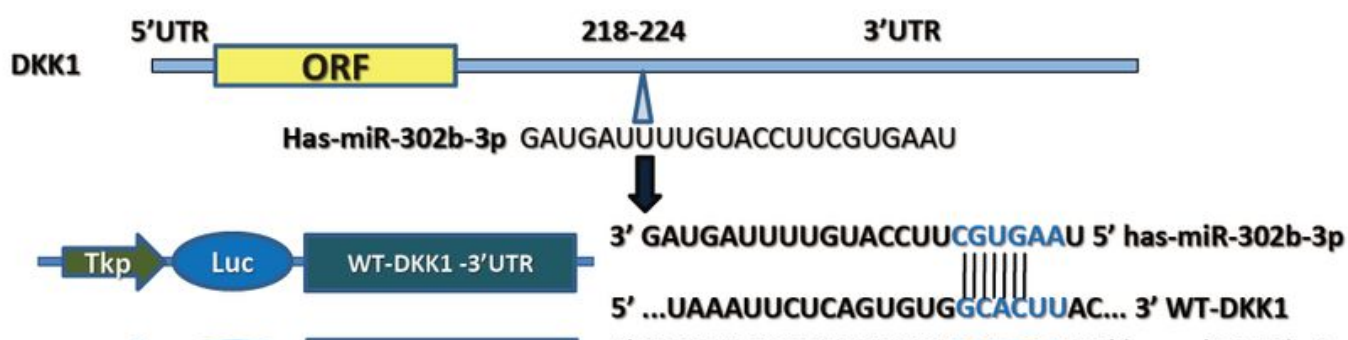

AUGUUUGACCUUCGUGAAU 5 ' has-miR-302b-3p

5'...UAAAUUCUCAGUGUGGCACUUAC... 3' WT-DKK1

Tkp LUC MUT-DKK1 -3'UTR

3' GAUGAUUUUGUACCUUCGUGAAU 5' has-miR-302b-3p

5'...UAAAUUCUCAGUGUGCGAGCGAC... 3' MUT-DKK1

B

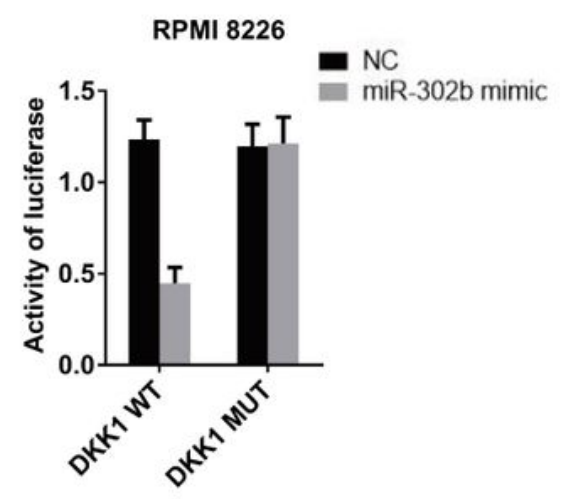

D

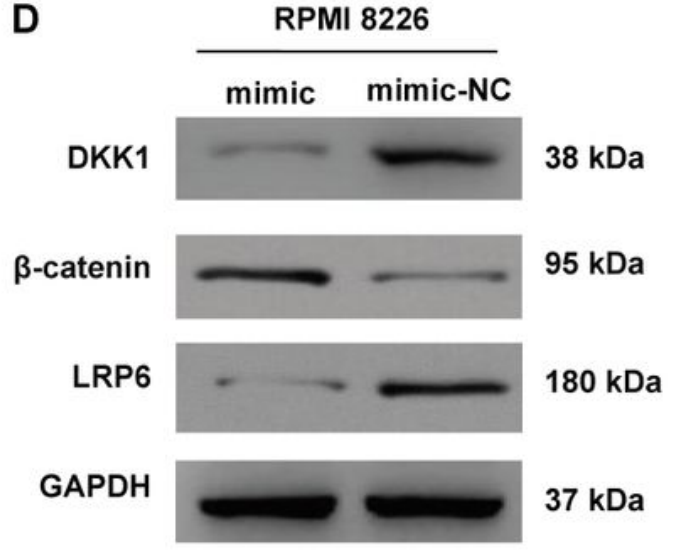

C

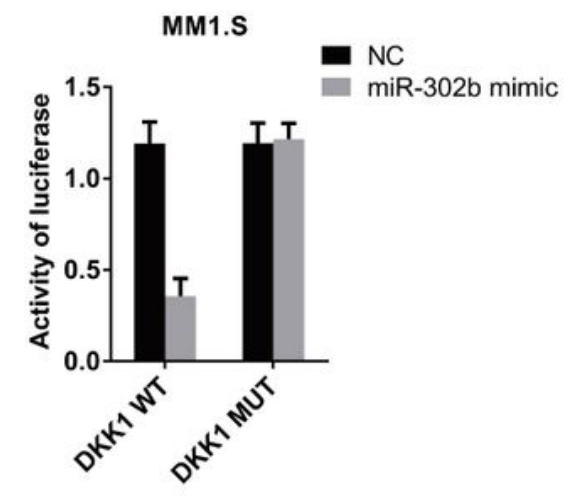

E

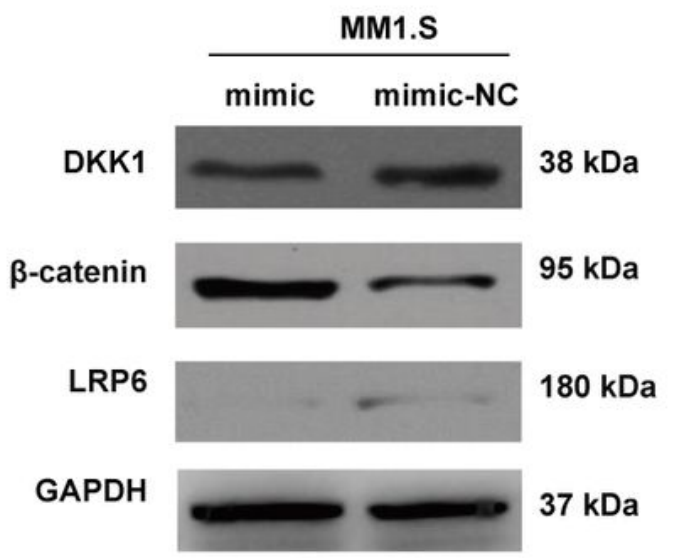

\section{Figure 3}

DKK1 is a direct target of miR-302b in MM cells. (A) Sequence alignment of predicted miR-302b binding sites within the DKK1 3'UTR and its mutated sequence for luciferase reporter assay. (B-C) Luciferase reporter assay was performed in RPMI 8226 cells and MM1.S cells that were co-transfected with miR302b mimics and reporter vectors containing DKK1 3'UTR or mutated DKK1 3'UTR. Relative luciferase activities are presented. ${ }^{*} \mathrm{P}<0.05$ vs. NC group. Data are presented as mean \pm SD from three separated 
experiments. (D-E) Expression of DKK1 in RPMI 8226 and MM1.S cells after the transfection of miR-302b mimics and mimics control.

A

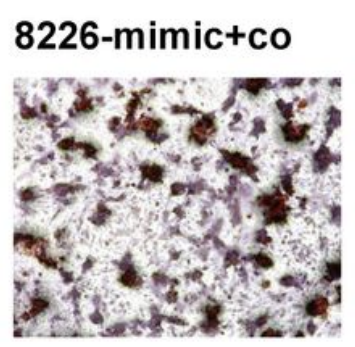

8226-mimic-NC+co

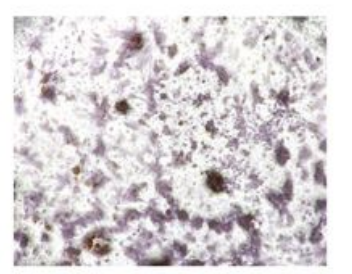

MM1.S-mimic+co MM1.S-mimic-NC+co
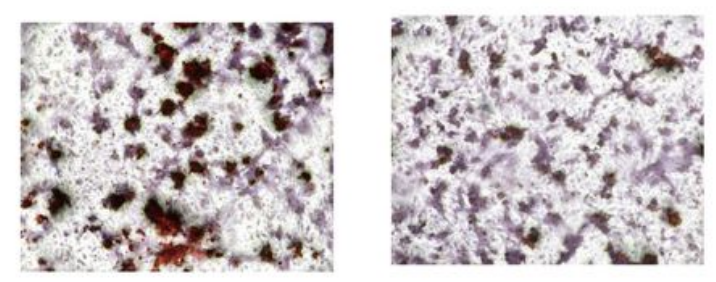

C

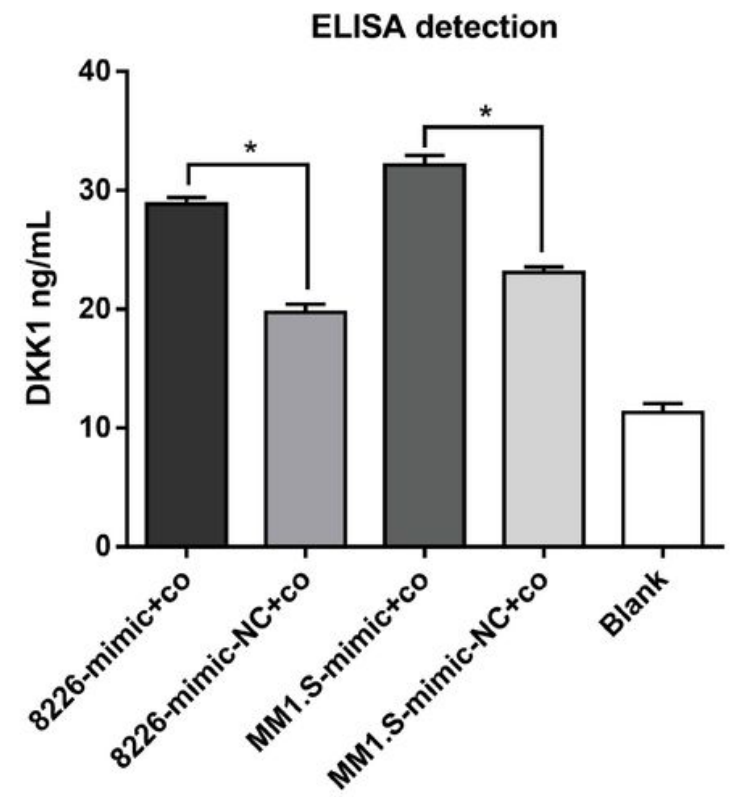

B

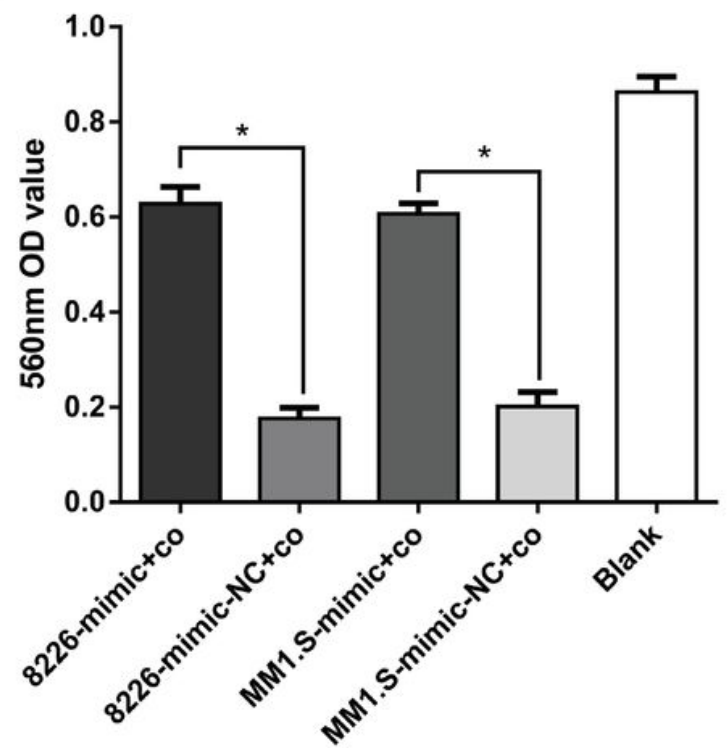

D

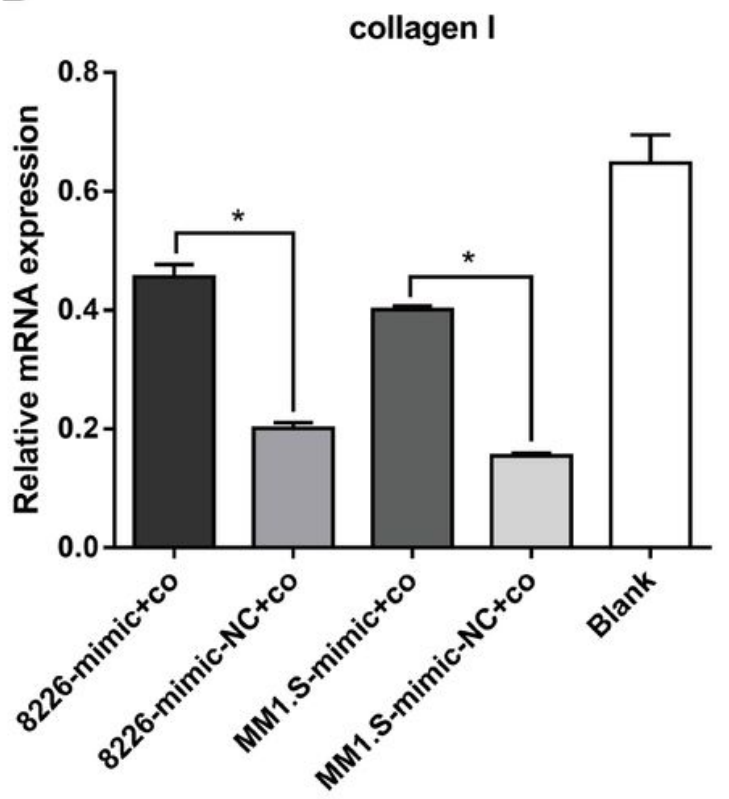

Figure 4

Effects of miR-302b on MM-induced osteogenic differentiation. (A) Alizarin red staining of MC3T3-E1 cells co-cultured with RPMI 8226 or MM1.S after transfection with miR-302b mimics and miR-302b mimics-NC respectively. (B) Quantification of the absorbance at $560 \mathrm{~nm}$ in (A) groups. (C) DKK1 protein content in the supernatant of MC3T3-E1 cells in (A) groups. (D) Collagen I mRNA expression of MC3T3E1 in (A) groups. Data are expressed as mean \pm SD. ${ }^{*}, \mathrm{P}<0.05$ 
A

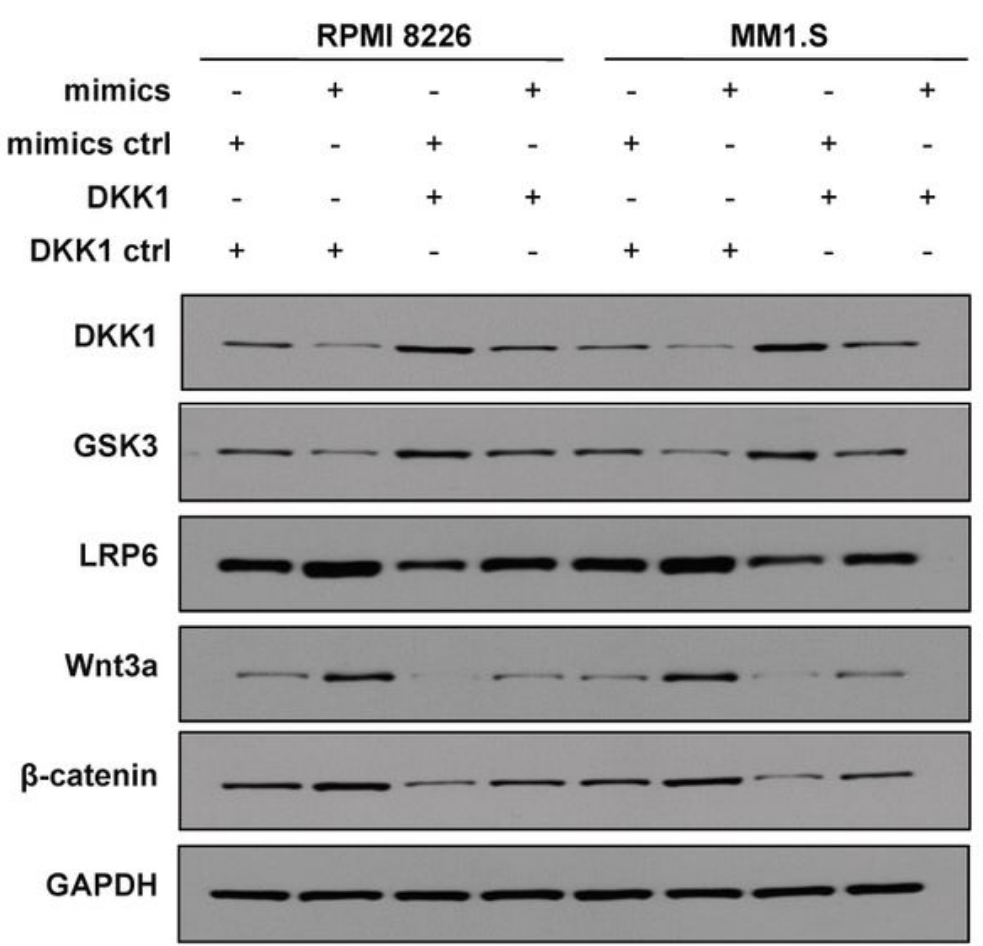

B

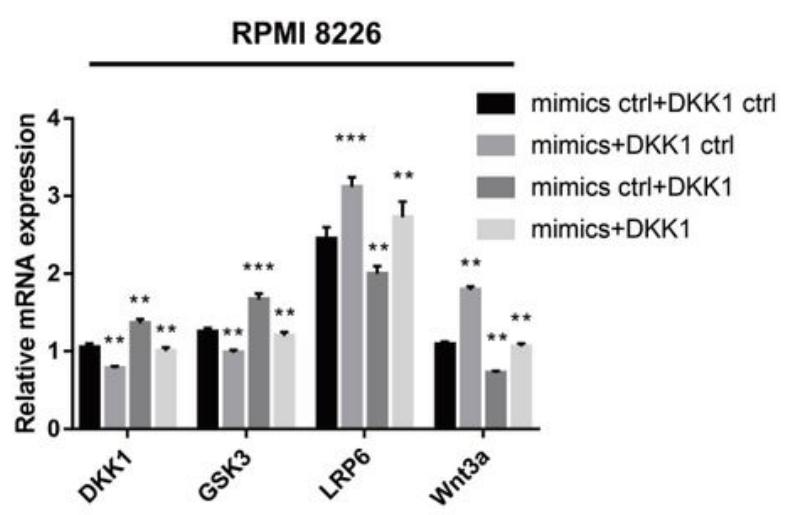

C

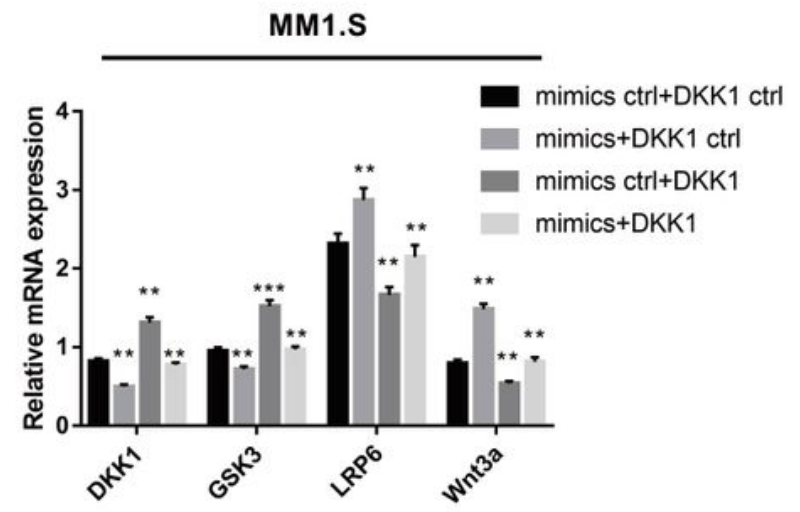

\section{Figure 5}

miR-302b inhibited Wnt/ $\beta$-catenin signaling pathway. (A) The expression levels of key proteins of the Wnt/ $\beta$-catenin pathway were detected by western blotting after transfection of RPMI 8226 and MM1.S cells after the transfection with miR-302b mimics (mimics)/miR-302b mimics-control (mimics ctrl) and DKK1 overexpression vectors (DKK1)/negative control vectors (DKK1 ctrl). (B-C) Expression of relative protein of the Wnt/ $\beta$-catenin pathway in RPMI 8226 and MM1.S cells. 
A

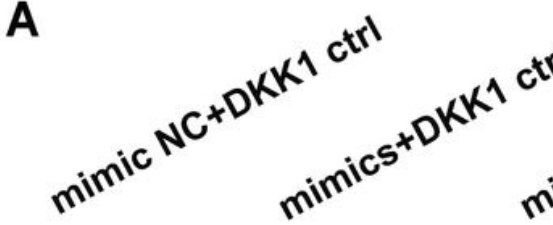

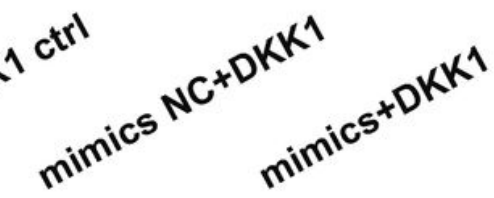
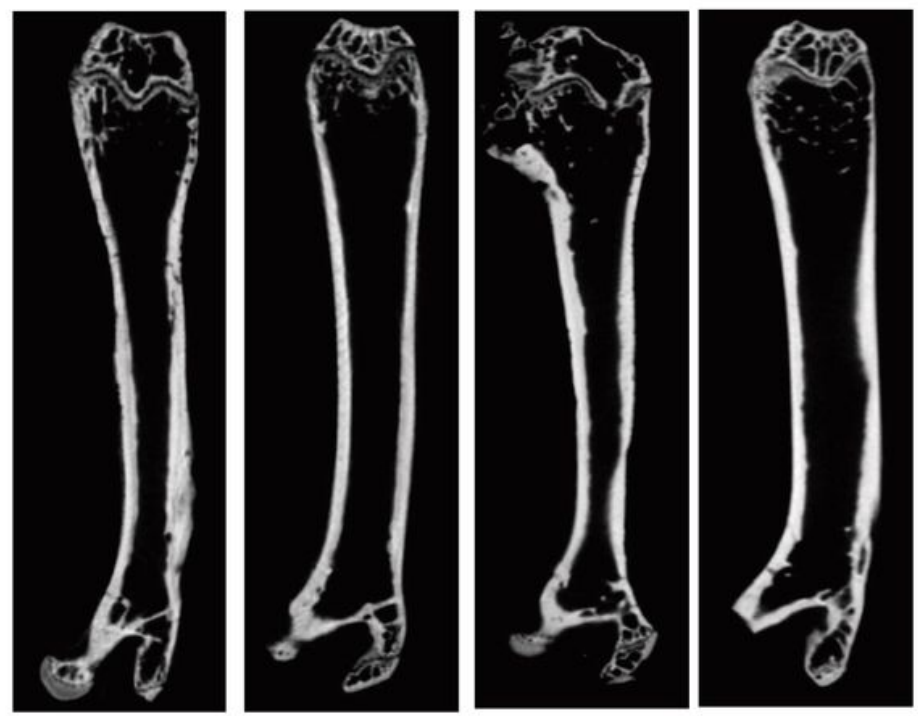

mimics NC+DKK1 ctrl

mimics+DKK1 ctrl
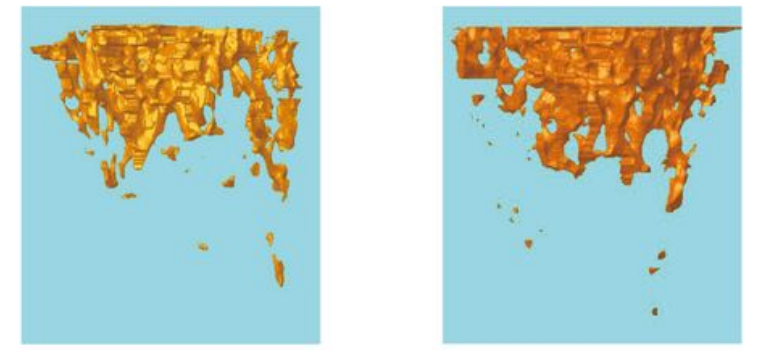

mimics NC+DKK1

mimics+DKK1
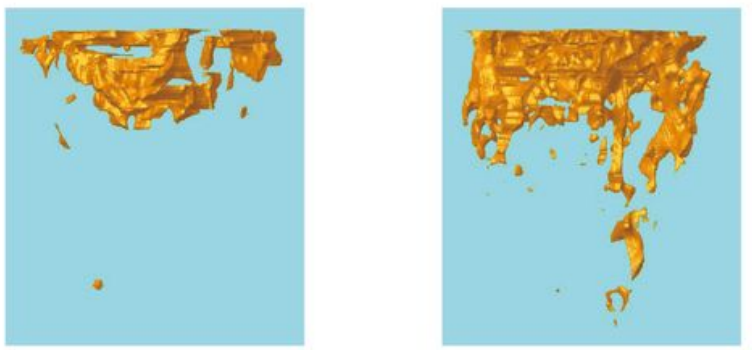

C Tb.N

BV/TV

Tb.Th

Tb.Pf
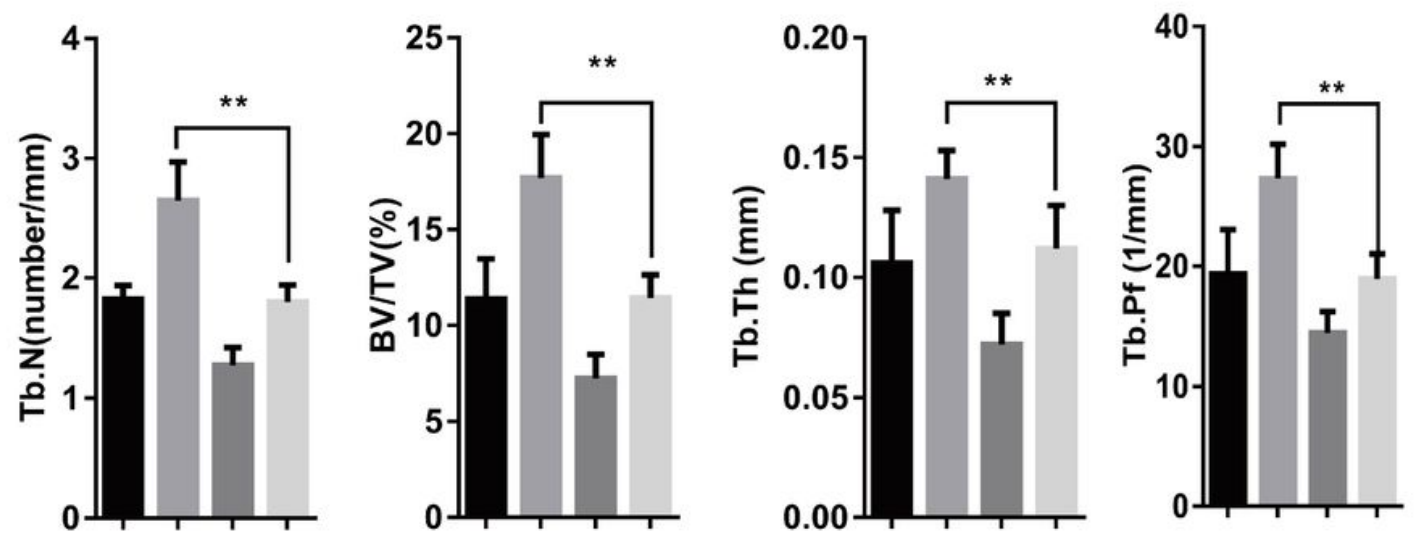

mimic NC+DKK1 ctrl

mimic+DKK1 ctrl

mimic NC+DKK1

mimic+DKK1

Figure 6

miR-302b delivery prevented MM-induced bone loss. (A-B) The femur images of micro-CT scanning of the NOD/SCID nude mice of each group. (C)Histomorphometric analysis of the trabecular bone in vertebrae, including BV/TV, Tb.Th, Tb.N, Tb.Pf Mean§SD, $n=8$ biologically in dependent samples, ${ }^{*} \mathrm{P}<0.01$ by twoway ANOVA. 
A

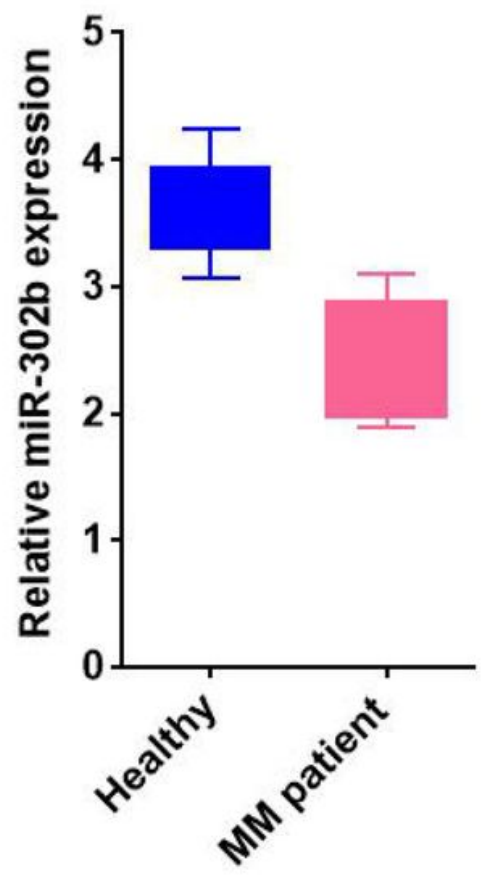

B

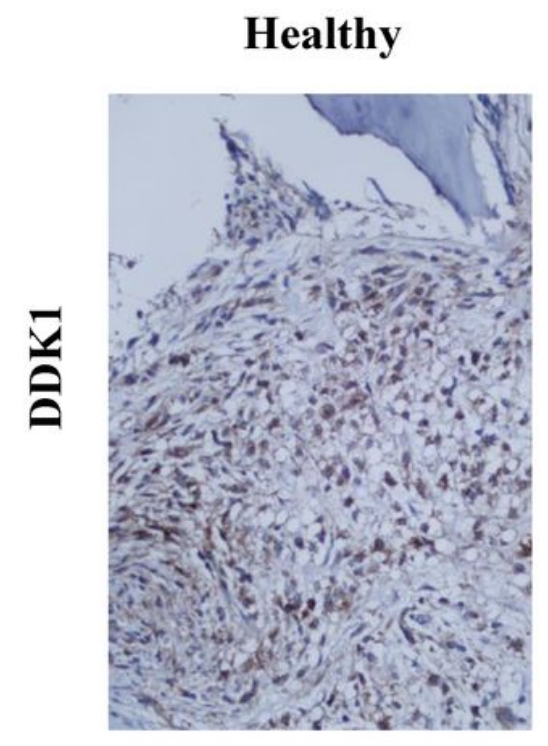

MM patient

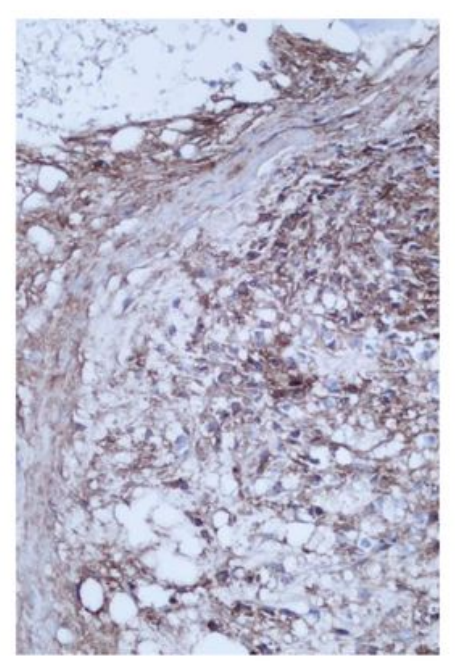

Figure 7

The miR-302b expression in clinical MM specimens. (A) Relative expression of miR-302b in MM tissues vs healthy group (B) Immunohistochemical staining of DKK1 in MM tissues and paired normal tissues. 